\title{
The Hidden Dangers of Obesity
}

\author{
Aida Sivro (University of Manitoba) \\ News Reporter (HSI 2011-2012)
}

Obesity has become a world-wide epidemic. In Canada, approximately one in four individuals are obese. It is well known that being overweight increases the risk of developing numerous health problems and chronic diseases, including cardiovascular disease, cancer, and type 2 diabetes. However, research is increasingly demonstrating adverse effects of obesity on the immune system especially in the context of influenza infections.

During the 2009 H1N1 influenza pandemic, obesity was identified as a risk factor for influenza-related morbidity and mortality. Data from the United States demonstrated that obesity was disproportionately represented in hospitalizations, intensive care admissions, and deaths. In fact, people with a BMI (body mass index) $>45$ had a 4.2 fold increase in the odds of death due to H1N1 independent of other risk factors. A similar study done at the University of Manitoba found that obesity was present in $33 \%$ of patients requiring intensive care admission. The reasons behind this

\section{...people with a BMI (body mass index) $>45$ had a 4.2 fold increased odds of death due to H1N1 independent of other risk factors.}

increase in morbidity and mortality due to influenza among obese individuals remain unknown. However, a number of factors have been theorized to contribute to the risk, including complications related to obesity in intensive care such as respiratory problems during general anaesthetic, the presence of obesity-related comorbidities such as diabetes, and the effects of obesity on the host immune response. ${ }^{1,2}$

An increase in visceral fat has been linked to the development of a low-grade systemic inflammatory state. Several studies performed in rodents have demonstrated that obese mice were more susceptible to morbidity and mortality due to influenza infection than normal mice as a

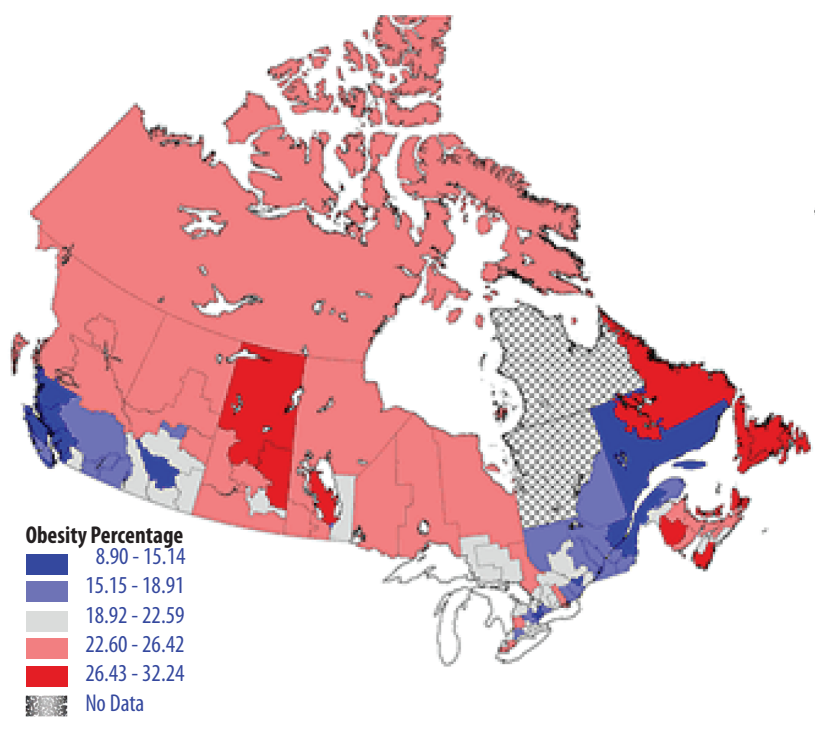

Adult obesity ( $\mathrm{BMI} \geq 30$ ) prevalence by health region, age and sex standardized to the 2005 Canadian population (25-64 years), Canadian Community Health Survey, 2005 (Source: http://www. phac-aspc.gc.ca/publicat/cdic-mcbc/30-1/ar_01-eng.php) [1]

result of changes in the innate immune response and the loss of function in influenza-specific immune cells. ${ }^{3}$ Despite recent advances in understanding the immune changes linked to excess visceral fat, the complex role obesity plays in immune deregulation remains an unexplored area in humans.

Obesity also appears to impair vaccine-induced immune responses in humans. ${ }^{4}$ In a study from the University of North Carolina, increased BMI was shown to be associated with a greater decline of protective antibodies over a 12-month period following influenza vaccination. In addition, impaired T-cell function, namely CD8+ T-cell activation and function, was observed in obese individuals compared to healthy controls. CD8+ T-cell responses specifically eliminate pathogens and protect against reinfection and are thought to be of paramount importance in the context of cross-protection against emerging new influenza strains. These findings suggest that obese individuals may remain susceptible to influenza infection even after vaccination. This is also consistent with previous research demonstrating a link between obesity and aberrant responses to tetanus 


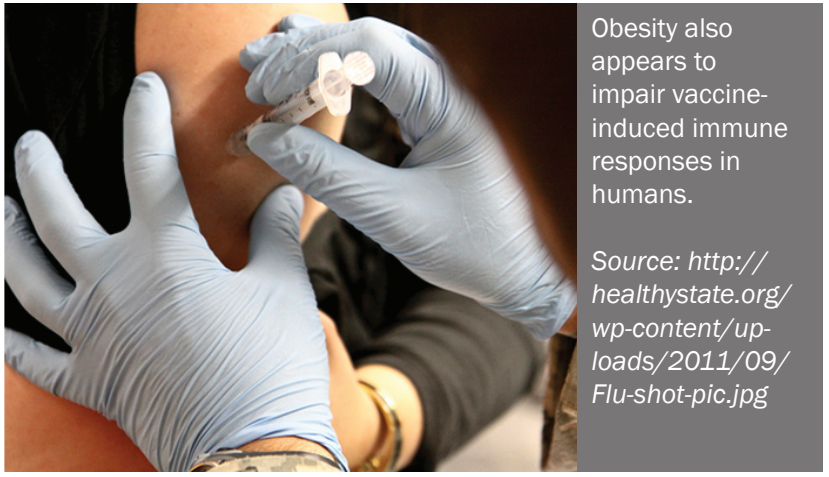

and hepatitis B vaccination. ${ }^{5,6}$ Overall, these findings have important implications, as current vaccine strategies may be less effective in achieving herd immunity in an increasingly obese population.

The combination of increasing rates of obesity with the continuing threat of new pandemic influenza strains could prove to be a serious public health threat. BMI may serve as an important measure to identify individuals at high risk of severe influenza. More research is needed in order to characterize the interaction between obesity and the human immune response, with emphasis on improving CD8+ responses generated by vaccines. Such research has the potential to affect prevention and treatment of infectious diseases in the future.

\section{References}

1. Louie, J., Acosta, M. \& Samuel, M. A Novel Risk Factor for a Novel Virus: Obesity and 2009 Pandemic Influenza A (H1N1). Clinical Infectious. (2011).

2. Morgan, O., Bramley, A., Fowlkes, A. \& Freedman, D. PLoS ONE: Morbid Obesity as a Risk Factor for Hospitalization and Death Due to 2009 Pandemic Influenza A(H1N1) Disease. PLoS ONE (2010).

3. Karlsson, E. \& Sheridan, P. Diet-Induced Obesity Impairs the T Cell Memory Response to Influenza Virus Infection. The Journal of Immunology (2010).

4. Sheridan, P. A. et al. Obesity is associated with impaired immune response to influenza vaccination in humans. Int J Obes Relat Metab Disord (2011). doi:10.1038/ijo.2011.208

5. Eliakim A, Schwindt C, Zaldivar F, Casali P, Cooper DM. Reduced tetanus antibody titers in overweight children. Autoimmunity 2006;39:137-141.

6. Weber DJ, Rutala WA, Samsa GP, Santimaw JE, Lemon SM. Obesity as a predictor of poor antibody response to hepatitis $B$ plasma vaccine. JAMA. $1985 ; 254: 3187-3189$

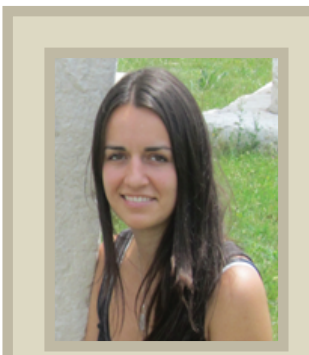

\section{Aida Sivro}

Aida Sivro is currently perusing a PhD in Medical Microbiology at the University of Manitoba. She is mainly interested in HIV immunology and the role host genetics plays in the susceptibility to HIV infection. Aida is also a trainee in the CIHR Infectious Diseases and Global Health Training Program (IID \& GHTP) 